\title{
RIEMANNIAN METRIC OF THE AVERAGED CONTROLLED KEPLER EQUATION
}

\author{
B. Bonnard, ${ }^{1}$ J.-B. Caillau, ${ }^{2}$ and R. Dujol ${ }^{2}$ \\ ${ }^{1}$ Institut de mathématiques de Bourgogne, UMR CNRS 5584, 9 avenue Alain Savary, F-21078 \\ Dijon,Bernard.Bonnard@univ-bourgogne.fr ${ }^{*},{ }^{2}$ ENSEEIHT-IRIT, UMR CNRS 5505, \\ 2 rue Camichel, F-31071 Toulouse, $\{$ caillau, dujol $\} @ n 7 . f r$
}

\begin{abstract}
A non-autonomous sub-Riemannian problem is considered: Since periodicity with respect to the independent variable is assumed, one can define the averaged problem. In the case of the minimization of the energy, the averaged Hamiltonian remains quadratic in the adjoint variable. When it is non-degenerate, a Riemannian problem and the corresponding metric can be uniquely associated to the averaged problem modulo the orthogonal group of the quadratic form. The analysis is applied to the controlled Kepler equation. Explicit computations provide the averaged Hamiltonian of the Kepler motion in the three-dimensional case. The Riemannian metric is given, and the curvature of a special subsytem is evaluated.
\end{abstract}

keywords: periodic sub-Riemannian problems, averaging, Riemannian metrics, minimum energy control, Kepler equation

\section{Introduction}

An elementary generalization of sub-Riemannian problems [6] is to take time-dependent vector fields: Instead of a linear in the control dynamics

$$
\dot{x}=\sum_{i=1}^{m} u_{i} f_{i}(x)
$$

where the $f_{i}$ 's generate a smooth distribution on the ambient manifold $X$ of dimension $n, n \geq m$, one considers vector fields $f_{i}(\theta, x)$, periodic with respect to the additional variable $\theta$. The dynamics of $\theta$ is known so that, up to a reparameterization of time, this amounts to dealing with non-autonomous vector fields. An approximation of the system is then provided by the averaged system:

\footnotetext{
*'The three authors are supported in part by the French Space Agency, contract 02/CNES/0257/00, and by the HYCON Network of Excellence, contract number FP6-IST-511368.
}

Please use the following format when citing this chapter:

Bonnard, B., Caillau, J.-B., and Dujol, R., 2006, in IFIP International Federation for Information Processing, Volume 202, Systems, Control, Modeling and Optimization, eds. Ceragioli, F., Dontchev, A., Furuta, H., Marti, K., Pandolfi, L., (Boston: Springer), pp. 79-89. 
Each vector field is averaged with respect to $\theta$ over a period. This approach is well known in optimal control, see [7] and [13], where the variable $\theta$ is regarded as the fast angular variable. Its application in celestial mechanics traces back to [12], and more recently to [8] in the case of orbit transfer problems with low thrust propulsion. Our aim is to analyze this problem, that is to contribute to the study of the controlled Kepler equation by means of averaging techniques initiated in [9]. The motivation is that the trajectories of the averaged system approximate those of the original problem which can only be computed numerically [5].

The first section is devoted to periodic sub-Riemannian problems in the previous sense. Since the averaging is performed on the Hamiltonian associated to the problem of minimization of the energy, we recall Pontryagin maximum principle before stating basic properties of the averaged Hamiltonian. The second section deals with the Riemannian structure defined by this Hamiltonian: When the quadratic form is non-degenerate on the cotangent bundle, a Riemannian metric can be canonically associated to the averaged problem modulo the action of the orthogonal group of the quadratic form. In the last section, we apply this approach to the controlled Kepler equation: The averaged Hamiltonian for minimum energy is explicitly computed in the three dimensional case, thus extending the results of [9]. Using an adapted change of coordinates, the metric corresponding to the two-dimensional problem is given in orthogonal form. As a first result in this Riemannian setting, the curvature of minimum energy transfers towards circular orbits is computed.

\section{Periodic sub-Riemannian problems}

Let $X$ be an $n$-dimensional manifold, and let $f_{i}(\theta, x), i=1, m$, be smooth vector fields parameterized by $\theta$ in $S^{1}$,

$$
f_{i}(\theta, x) \in T_{x} X, \theta \in S^{1}, x \in X .
$$

The corresponding periodic sub-Riemannian dynamics is defined by

$$
\begin{aligned}
\dot{x} & =\sum_{i=1}^{m} u_{i} f_{i}(\theta, x) \\
\dot{\theta} & =g_{0}(\theta, x)+g_{1}(\theta, x, u) .
\end{aligned}
$$

The two functions $g_{0}$ and $g_{1}$ are smooth, $g_{0}$ positive, and $g_{1}$ is assumed to be linear in $u$. The broader class of sub-Riemannian systems with drift [5] may provide examples of such dynamics. Indeed, if $\dot{x}=f_{0}(x)+\sum_{i=1}^{m} u_{i} f_{i}(x)$ with $f_{0}=g_{0} \partial / \partial x_{n}$, the system falls into the previous class with $\theta=x_{n}$ provided periodicity of the $f_{i}$ 's with respect to $x_{n}$ holds. As shall be stated in section 3 , this is indeed the case for the controlled Kepler equation. 
Standard performance indexes for (1-2) are minimum time, minimum length,

$$
\int_{0}^{t_{f}} \sqrt{\left|u_{1}\right|^{2}+\cdots+\left|u_{m}\right|^{2}} d t \rightarrow \min
$$

or minimum energy :

$$
\int_{0}^{t_{f}}\left(\left|u_{1}\right|^{2}+\cdots+\left|u_{m}\right|^{2}\right) d t \rightarrow \min .
$$

For the problem to make sense, one usually has to add a bound constraint on the control, $|u| \leq \varepsilon$. The choice of the finite- dimensional norm $|$.$| is of course$ crucial (the control set may not even be smooth). Since Maupertuis' principle does not hold here, minimizing the length-that is the $\mathrm{L}^{1}$-norm of the controlmay give rise to intricate optimal control problems (see for instance [10] in the case of Kepler equation). We focus here on the minimization of the energy, that is on the optimization of the $\mathrm{L}^{2}$-norm of the control, the final time being fixed. In this particular case, we first relax the problem by dropping the bound on the control. Indeed, the underlying idea is that, for a given positive $\varepsilon$, the constraint will be automatically fulfilled for a big enough fixed final time. Hence, treating the control as a small quantity, it is natural to do the feedback $u=\varepsilon v$ and to reparameterize the trajectories by $\theta$ :

$$
\frac{d x}{d \theta}=\frac{\varepsilon}{g_{0}(\theta, x)+\varepsilon g_{1}(\theta, x, v)} \sum_{i=1}^{m} v_{i} f_{i}(\theta, x)
$$

The criterion becomes

$$
\varepsilon^{2} \int_{\theta_{0}}^{\theta_{f}}\left(\left|v_{1}\right|^{2}+\cdots+\left|v_{m}\right|^{2}\right) \frac{d \theta}{g_{0}(\theta, x)+\varepsilon g_{1}(\theta, x, v)}
$$

and Pontryagin maximum principle tells us that optimal trajectories are projection on $X$ of the integral curves of the following Hamiltonian defined on the cotangent bundle $T^{*} X$ :

$$
H(\theta, x, p, v)=\frac{\varepsilon}{g_{0}(\theta, x)+\varepsilon g_{1}(\theta, x, v)}\left(p^{0} \varepsilon|v|^{2}+\sum_{i=1}^{m} v_{i} P_{i}(\theta, x, p)\right) .
$$

Here before, $p^{0}$ is a nonpositive constant, $p$ is the adjoint state to $x$ and belongs to the cotangent space $T_{x}^{*} X$, and the $P_{i}$ 's are the Poincaré coordinates or Hamiltonian lifts of the vector fields,

$$
P_{i}(\theta, x, p)=\left\langle p, f_{i}(\theta, x)\right\rangle, i=1, m \text {. }
$$

We consider the so-called normal case, $p^{0}$ negative: For obvious homogeneity reasons, we use the normalization $p^{0}=-1 / 2 \varepsilon$. Consequently, up to first order 
in $\varepsilon$, we have the following approximation of $H$ :

$$
\begin{aligned}
H(\theta, x, p, v) & =\frac{\varepsilon}{g_{0}}\left(1-\varepsilon g_{1} / g_{0}+\cdots\right)\left(-\frac{1}{2}|v|^{2}+\sum_{i=1}^{m} v_{i} P_{i}(\theta, x, p)\right) \\
& =\frac{\varepsilon}{g_{0}(x, \theta)}\left(-\frac{1}{2}|v|^{2}+\sum_{i=1}^{m} v_{i} P_{i}(\theta, x, p)\right)+o(\varepsilon) .
\end{aligned}
$$

According to the maximum principle, the optimal control maximizes $H$ so the maximized first order approximation of the Hamiltonian, which we still denote $H$, is the true ${ }^{1}$ Hamiltonian function

$$
H(\theta, x, p)=\frac{1}{2 g_{0}(x, \theta)} \sum_{i=1}^{m} P_{i}^{2}(\theta, x, p)
$$

where, for the sake of simplicity, we have dropped the multiplicative factor $\varepsilon$. This Hamiltonian is clearly invariant with respect to feedbacks $v=R(\theta, x) v^{\prime}$, $R(\theta, x)$ in $\mathrm{SO}(m)$.

The averaged Hamiltonian is

$$
\bar{H}(x, p)=\frac{1}{2 \pi} \int_{0}^{2 \pi} H(\theta, x, p) d \theta .
$$

Under mild assumptions, the integral curves of the averaged system converge uniformly towards those of the original system, see for instance [1, chap. 10]. The function $H(\theta, x, p)$ is a non-negative quadratic form in $p$, possibly degenerate, with coefficients parameterized respectively by $\theta$ and $x$. We denote by $w(\theta, x)$ this form. Since the integral is positive and linear, the following holds.

LEMMA 1 The averaged Hamiltonian also defines a non-negative quadratic form in $p$, denoted by $w(x)$. Moreover,

$$
\operatorname{Ker} w(x)=\bigcap_{\theta \in S^{1}} \operatorname{Ker} w(\theta, x) .
$$

According to this lemma, we can only expect the rank to increase. An interpretation is that the oscillations of the fast variable $\theta$ generate new control directions, namely brackets of the original vector fields. We will assume in the sequel that $w(x)$ is non- degenerate.

\section{Riemannian structure of the averaged problem}

Let $w$ be a smooth function on $X$ such that, for any point $x, w(x)$ defines a positive definite quadratic form on the fiber $T_{x}^{*} X$. Then, $w$ can be represented by its polar form which is a two-times covariant symmetric tensor. In coordinates,

$$
w(x)=\sum_{i, j=1}^{m} w_{i j}(x) \frac{\partial}{\partial x_{i}} \otimes \frac{\partial}{\partial x_{j}} .
$$


The matrix $W(x)=\left(w_{i j}(x)\right)_{i j}$ belongs to the cone of real positive definite symmetric matrices, $\operatorname{Sym}_{+}(n, \mathbf{R})$. This tensor defines a Riemannian structure [11] on the fiber space $T^{*} X$. The question is: Does it arise from a Riemannian problem on $X$ ? That is, is the Hamiltonian $H(x, p)=1 / 2 \sum_{i, j=1}^{m} w_{i j}(x) p_{i} p_{j}$ associated with a control problem

$$
\int_{0}^{t}\left(\left|u_{1}\right|^{2}+\cdots+\left|u_{n}\right|^{2}\right) d t \rightarrow \min
$$

and $\dot{x}=u_{1} f_{1}(x)+\cdots+u_{n} f_{n}(x)$ (where there are $m=n$ vector fields and controls)? Now, the answer is obviously positive since one has just to write $H$ as a sum of squares, using a change of adjoint variable of the type $p=q^{t} A(x)$,

$$
H(x, p)=\frac{1}{2} \sum_{i=1}^{n} P_{i}^{2}(x, p)
$$

with $P_{i}(x, p)=\left\langle p, f_{i}(x)\right\rangle$ (the last equalities defining so the $f_{i}$ 's). Indeed, the Hamiltonian is a quadratic form in $p$ parameterized by $x$, so it can be written as a sum of independent linear forms in $p$, using for instance Gauss algorithm. To this end, let us recall the (right) action of the linear group $\operatorname{GL}(n, \mathbf{R})$ on $\operatorname{Sym}_{+}(n, \mathbf{R})$. For $A$ in $\mathrm{GL}(n, \mathbf{R})$, one defines

$$
A \cdot M={ }^{t} A M A, M \in \operatorname{Sym}_{+}(n, \mathbf{R}) \text {. }
$$

Given $M$ in $\operatorname{Sym}_{+}(n, \mathbf{R})$, we look for $A$ such that $A \cdot M=I$ : The relevant set is hence the set of $M$-orthonormal matrices. The isotropy group of $M$ is the orthogonal group $\mathrm{O}(M)$ of matrices $O$ such that

$$
{ }^{t} O M O=M
$$

Therefore, two matrices $A$ and $B$ are both $M$-orthogonal if and only if $B^{-1}$. $(A \cdot M)=\left(A B^{-1}\right) \cdot M=M$, that is if $A B^{-1}$ belongs to $\mathrm{O}(M)$. The set of $M-$ orthonormal matrices is thus in one-to-one correspondance with the orthogonal group, and $M$ is diagonalized to identity by a unique element of the quotient set $\mathrm{GL}(n, \mathbf{R}) / \mathrm{O}(M)$.

Let now $\mathrm{O}(w(x))$ be the orthogonal group generated by the quadratic form $w(x)$, and let $A(x)$ be in $\mathrm{GL}(n, \mathbf{R}) / \mathrm{O}(w(x))$ : The Hamiltonian writes

$$
H(x, p)=\frac{1}{2}|q|^{2}=\frac{1}{2}\left|p^{t} A(x)^{-1}\right|^{2}
$$

and comes from the Riemannian problem with dynamics $\dot{x}={ }^{t} A(x)^{-1} u$. Eventually, $|u|^{2}$ is equal to $\left|{ }^{t} A(x) \dot{x}\right|^{2}$ and the Riemannian metric is $d s^{2}=$ 
$\sum_{i j=1}^{n} g_{i j} d x_{i} d x_{j}$ where $G(x)=\left(g_{i j}(x)\right)_{i j}$ is the positive definite symmetric matrix $\left(A^{t} A\right)(x)$. The following proposition summarizes the computations.

Proposition 1 The Riemannian metric associated to the Riemannian structure on the cotangent bundle is unique modulo the action on positive definite symmetric matrices of the quadratic form defining the structure.

Proof. Let $M$ be a positive definite symmetric matrix. If both $A$ and $B$ are $M$-orthonormal, there is $O$ in $\mathrm{O}(M)$ such that $A=O B$. Then $A^{t} A=O \cdot\left(B^{t} B\right)$ for the (left) action of $O(M)$ on $\operatorname{Sym}_{+}(n, \mathbf{R}), O \cdot Y=O Y^{t} O$, and $A^{t} A$ and $B^{t} B$ belong to the same orbit.

We end this section by recalling the effect of a change of variables in $X$ on the tensor $w$. Indeed, it is not realistic to look for coordinates that would trivialize $w$ to $\sum_{i=1}^{n}\left(\partial / \partial y_{i}\right)^{2}$ since then, the associated metric would be flat. A less strong requirement may be to find such coordinates that diagonalize the quadratic form,

$$
w(y)=\sum_{i=1}^{n} d_{i}(y)\left(\frac{\partial}{\partial y_{i}}\right)^{2}, d_{i}(y)>0 .
$$

In this case, the associated metric on $X$ is orthogonal [2],

$$
d s^{2}=\sum_{i=1}^{n} \frac{d y_{i}^{2}}{d_{i}(y)}
$$

Given $M$ in $\operatorname{Sym}_{+}(n, \mathbf{R})$, this amounts to finding $M$-orthogonal matrices. As a consequence of Sylvester's law of inertia, two matrices $A$ and $B$ are $M-$ orthogonal if and only if there is a scaling, that is an element of $\left(\mathbf{R}_{+}^{*}\right)^{n}$ (defining a diagonal matrix $S$ with positive entries), such that $S \cdot(A \cdot M)=B \cdot M$. In other words, $M$-orthogonal matrices are in one-to-one correspondance with elements of the direct product $\mathrm{O}(M) \times\left(\mathbf{R}_{+}^{*}\right)^{n}$.

If $x=\varphi(y)$ is a change of coordinates on $X$, the new adjoint state $q$ and tensor matrix $U$ verify:

$$
p=q d \varphi(y)^{-1}, \quad W(x)={ }^{t} d \varphi(y) \cdot U(y) .
$$

Accordingly, one gets an orthogonal metric on $X$ if and only if $\varphi$ is such that $d \varphi$ is $(W \circ \varphi)^{-1}$-orthogonal. As we shall see now in the last section, it turns out that such a change of coordinates is available in the Kepler case.

\section{Application to the controlled Kepler equation}

We briefly state the control problem, see for instance [5] for a detailed exposition. The Kepler equation describes the motion of a body in a central field and can be normalized to

$$
\ddot{q}=-\frac{q}{|q|^{3}}+\gamma
$$


where $|q|^{2}=\left|q_{1}\right|^{2}+\left|q_{2}\right|^{2}+\left|q_{3}\right|^{2}$, $q$ being the position vector in $\mathbf{R}^{3}$ and $\gamma$ the control. Several sets of coordinates describing the geometry of the osculating conic are available. The dynamics also depends on the local frame chosen to express the control. In contrast with $[8,9]$ where the so-called tangential-normal frame is used, we use the feedback-invariance of the maximized Hamiltonian and prefer to write down the equations in the radial-orthoradial frame for reasons that will be made clear in the next paragraph: $\gamma=u_{1} f_{1}+u_{2} f_{2}+u_{3} f_{3}$ with $f_{1}=q /|q|, f_{2}=f_{3} \times f_{1}$ and $f_{3}=q \times \dot{q} /|q \times \dot{q}|$. The state is described by five equinoctial elements, for instance $x=(P, e, h)$ where $P$ is the semilatus rectum, $e=\left(e_{x}, e_{y}\right)$ the eccentricity vector, $h=\left(h_{x}, h_{y}\right)$ the inclination vector, and by an angle, the true longitude $l$. We restrict the problem to the manifold $X$ of elliptic trajectories,

$$
X=\{(P, e, h) \mid P>0 \text { and }|e|<1\}
$$

so that the vector fields are smooth on $S^{1} \times X$ and define a periodic subRiemannian problem as in section 34 :

$$
\begin{aligned}
& f_{1}=\sqrt{P}\left(\sin l \frac{\partial}{\partial e_{x}}-\cos l \frac{\partial}{\partial e_{y}}\right) \\
& f_{2}=\sqrt{P}\left[\frac{2 P}{W} \frac{\partial}{\partial P}+\left(\cos l+\frac{e_{x}+\cos l}{W}\right) \frac{\partial}{\partial e_{x}}+\left(\sin l+\frac{e_{y}+\sin l}{W}\right) \frac{\partial}{\partial e_{y}}\right] \\
& f_{3}=\frac{\sqrt{P}}{W}\left(-Z e_{y} \frac{\partial}{\partial e_{x}}+Z e_{x} \frac{\partial}{\partial e_{y}}+\frac{C \cos l}{2} \frac{\partial}{\partial h_{x}}+\frac{C \sin l}{2} \frac{\partial}{\partial h_{y}}\right)
\end{aligned}
$$

where

$$
\begin{aligned}
W & =1+e_{x} \cos l+e_{y} \sin l \\
Z & =h_{x} \sin l-h_{y} \cos l \\
C & =1+|h|^{2} .
\end{aligned}
$$

The variation of the angle is $i=g_{0}(l, x)+g_{1}(l, x, u)$ with

$$
g_{0}=\frac{W^{2}}{P^{3 / 2}} \quad g_{1}=\sqrt{P} \frac{Z}{W} u_{3} .
$$

An important remark is that, because there is some decoupling between these vector fields, the two-dimensional Kepler problem is obtained by letting $h=0$ in the previous equations. We start with the computation on this subproblem.

Applying the process described in section 34, one gets the maximized first order approximation of the Hamiltonian, $H=\left(P_{1}^{2}+P_{2}^{2}\right) / 2$ with

$$
\begin{aligned}
& P_{1}=\frac{P^{5 / 4}}{W}\left(p_{e_{x}} \sin l-p_{e_{y}} \cos l\right) \\
& P_{2}=\frac{P^{5 / 4}}{W}\left[p_{P} \frac{2 P}{W}+p_{e_{x}}\left(\cos l+\frac{e_{x}+\cos l}{W}\right)+p_{e_{y}}\left(\sin l+\frac{e_{y}+\sin l}{W}\right)\right] .
\end{aligned}
$$


Hence, the computation of the averaged has the complexity of integrating terms of the form $P(\cos l, \sin l) / W^{k}$, where $P$ is a polynomial and $k$ and integer comprised between 2 and 4 . Since

$$
\int_{0}^{2 \pi} \frac{P(\cos l, \sin l)}{W^{k}} d l=\int_{S^{1}} \frac{P(z / 2+1 / 2 z, z / 2 i-1 / 2 i z)}{W^{k}} \frac{d z}{i z}
$$

the averaged is evaluated by computing the residues of the integrands. Since $W=\left(\bar{e} z^{2}+2 z+e\right) / 2 z$ (we use the complex eccentricity $e=e_{x}+i e_{y}$ ), we have two poles,

$$
z=\frac{-1 \pm \sqrt{1-|e|^{2}}}{\bar{e}}
$$

The product of the poles is $e / \bar{e}$ so it has modulus one, and $z_{1}=(-1-$ $\left.\sqrt{1-|e|^{2}}\right) / \bar{e}$ clearly does not belong to the unit disk: The only pole to consider is $z_{2}=\left(-1+\sqrt{1-|e|^{2}}\right) / \bar{e}$. In contrast, if one uses the tangential-normal frame as in [9], $W$ is replaced by $W\left(1+2 e_{x} \cos l+2 e_{y} \sin l+|e|^{2}\right)$, and two poles among the four are to be taken into account.

An inspection of the Hamiltonian shows that the following averages have to be computed, for which we give the results:

$$
\begin{aligned}
& \overline{1 / W^{2}}=\delta^{3}
\end{aligned}
$$

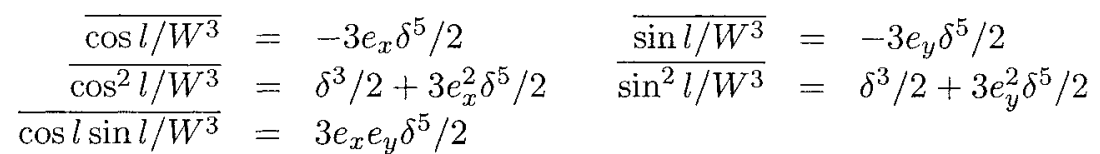

$$
\begin{aligned}
& \begin{aligned}
\overline{1 / W^{4}} & =\left(2+3|e|^{2}\right) \delta^{7} / 2 \\
\frac{\cos l / W^{4}}{\cos ^{2} l / W^{4}} & =-e_{x}\left(4+|e|^{2}\right) \delta^{7} / 2 \quad \overline{\sin l / W^{4}}=-e_{y}\left(4+|e|^{2}\right) \delta^{7} / 2 \\
\frac{\sin ^{2} l / W^{4}}{\cos l \sin l / W^{4}} & =5 e_{x} e_{y} \delta^{7} / 2
\end{aligned}
\end{aligned}
$$

with $\delta=1 / \sqrt{1-|e|^{2}}$. Substituing these expressions, we get the averaged Hamiltonian

$$
\begin{aligned}
\vec{H}(x, p) & =\frac{P^{5 / 2}}{4\left(1-|e|^{2}\right)^{5 / 2}}\left[4 p_{P}^{2} P^{2}\left(-3+\frac{5}{1-|e|^{2}}\right)\right. \\
& +p_{e_{x}}^{2}\left(5\left(1-|e|^{2}\right)+e_{y}^{2}\right)+p_{e_{y}}^{2}\left(5\left(1-|e|^{2}\right)+e_{x}^{2}\right) \\
& \left.-20 p_{P} p_{e_{x}} P e_{x}-20 p_{P} p_{e_{y}} P e_{y}-2 p_{e_{x}} p_{e_{y}} e_{x} e_{y}\right] .
\end{aligned}
$$

At this point, we take advantage of the computation in [9] and make the following change of variables:

$$
P=\frac{1-\rho^{2}}{n^{2 / 3}}
$$




$$
e_{x}=\rho \cos \theta \quad e_{y}=\rho \sin \theta
$$

where $n$ is the so-called mean movement [14]. Using 4), we obtain

$$
\vec{H}=\frac{1}{4 n^{5 / 3}}\left[18 n^{2} p_{n}^{2}+5\left(1-\rho^{2}\right) p_{\rho}^{2}+\left(5-4 \rho^{2}\right) \frac{p_{\theta}^{2}}{\rho^{2}}\right] \text {. }
$$

Up to a scalar, this is the result that was obtained by symbolic machine computation in [9]. However, the complexity of the computation did not allow the authors to tackle the three-dimensional problem whereas we shall be able to do so, here. Let us write before the Riemannian metric of the two-dimensional Kepler problem in orthogonal form:

$$
d s^{2}=\frac{1}{9 n^{1 / 3}} d n^{2}+\frac{2 n^{5 / 3}}{5\left(1-\rho^{2}\right)} d \rho^{2}+\frac{2 n^{5 / 3}}{5-4 \rho^{2}} \rho^{2} d \theta^{2} .
$$

The three-dimensional case has the same complexity: Indeed, the Hamiltonian is $H=\left(P_{1}^{2}+P_{2}^{2}+P_{3}^{2}\right) / 2$ with

$$
P_{3}=\frac{P^{5 / 4}}{W^{2}}\left(-Z p_{e_{x}} e_{y}+Z p_{e_{y}} e_{x}+C / 2 p_{h_{x}} \cos l+C / 2 p_{h_{y}} \sin l\right)
$$

and $P_{1}, P_{2}$ unchanged. Hence, the previous averaging computations allow us to conclude: Extending the change of coordinates to $h$ according to $h_{x}=\sigma \cos \Omega$, $h_{y}=\sigma \sin \Omega$, we get

$$
\begin{aligned}
\bar{H} & =\frac{1}{4 n^{5 / 3}}\left[18 n^{2} p_{n}^{2}+5\left(1-\rho^{2}\right) p_{\rho}^{2}+\left(5-4 \rho^{2}\right) \frac{p_{\theta}^{2}}{\rho^{2}}\right] \\
& +\frac{1}{4 n^{5 / 3}} \frac{\left(1+\sigma^{2}\right)^{2}}{4} \frac{1+4 \rho^{2}}{1-\rho^{2}}\left(\cos \omega p_{\sigma}+\sin \omega \frac{p_{\theta \Omega}}{\sigma}\right)^{2} \\
& +\frac{1}{4 n^{5 / 3}} \frac{\left(1+\sigma^{2}\right)^{2}}{4}\left(-\sin \omega p_{\sigma}+\cos \omega \frac{p_{\theta \Omega}}{\sigma}\right)^{2}
\end{aligned}
$$

where $\omega=\theta-\Omega$ is the angle of the pericenter and where

$$
p_{\theta \Omega}=\frac{2 \sigma^{2}}{1+\sigma^{2}} p_{\theta}+p_{\Omega}
$$

We conclude the exposition by a preliminary computation of curvature. In the two-dimensional case, one can restrict the metric to $\{\theta=0\}$. The associated trajectories are, for instance, those reaching a circular orbit. On this submanifold of dimension two, the metric is

$$
d s^{2}=\frac{1}{9 n^{1 / 3}} d n^{2}+\frac{2 n^{5 / 3}}{5\left(1-\rho^{2}\right)} d \rho^{2}
$$


and the following holds.

Proposition 2 The curvature is zero.

Proof. In orthogonal coordinates, the Gaussian curvature is

$$
K=-\frac{1}{2 \sqrt{g}}\left[\frac{\partial}{\partial n}\left(\frac{1}{\sqrt{g}} \frac{\partial g_{11}}{\partial n}\right)+\frac{\partial}{\partial \rho}\left(\frac{1}{\sqrt{g}} \frac{\partial g_{22}}{\partial \rho}\right)\right]
$$

with $g=g_{11} g_{22}$, whence the result.

As a result, the metric is locally isomorphic to $d s^{2}=d x^{2}+d y^{2}$. Actually, we prove in [3] that the result is global and that we can find coordinates in which the two-dimensional subsystem is flat. The insight on the control of the Kepler equation provided by this Riemannian point of view will be developed in forthcoming papers. See for instance [4] for a preliminary evaluation of Riemannian balls of the two-dimensional Kepler motion.

\section{Notes}

1. True in the sense that it is not parameterized by the control anymore.

\section{References}

[1] V. I. Arnold. Mathematical Methods of Classical Mechanics. Springer-Verlag, New-York, 1978.

[2] M. Berger, B. Gostiaux. Géométrie différentielle. Presses Universitaires de France, Paris, 1981.

[3] B. Bonnard, J.-B. Caillau. Riemannian metric of the averaged energy minimization problem in orbital transfer with low thrust. Submitted to Annales de l'Institut Henri Poincaré.

[4] B. Bonnard, J.-B. Caillau, R. Dujol. Averaging and optimal control of elliptic Keplerian orbits with low propulsion. Submitted to 13ih IFAC Workshop on Control Applications of Optimisation, Paris, April 2006.

[5] B. Bonnard, J.-B. Caillau, E. Trélat. Geometric optimal control of elliptic Keplerian orbits. Disc. and Cont. Dyn. Sysi. Series B 5(4):929-956, 2005.

[6] B. Bonnard, M. Chyba. Singular trajectories and their role in optimal control. Math. and Applications 40, Springer Verlag, Paris, 2003.

[7] F. Chaplais. Averaging and deterministic optimal control. SIAM J. Control and Opt. 25(3):767-780, 1987.

[8] R. Epenoy, S. Geffroy. Optimal low-thrust transfers with constraints. Generalization of averaging techniques. Acta Astronautica 41(3):133-149, 1997.

[9] S. Geffroy. Les techniques de moyennisation en contrôle optimal. Application aux transferts orbitaux à poussée continue. Master thesis, ENSEEIHT, Institut National Polytechnique de Toulouse, 1994.

[10] J. Gergaud, T. Haberkorn. Homotopy method for minimum consumption orbit transfer problem. ESAIM Control Opt. and Calc. of Var, to appear.

[11] C. Godbillon. Géométrie différentielle et mécanique analytique. Hermann, Paris, 1985. 
[12] J. Kevorkian, J. D. Cole. Perturbation methods in applied mathematics. Springer Verlag, New-York, 1981.

[13] S. G. Peng. Analyse asymptotique et problème homogénéisé en contrôle optimal avec vibrations rapides. SIAM J. Control and Opt. 27(4):673-696, 1989.

[14] O. Zarrouati. Trajectoires spatiales. CNES-Cepadues, Toulouse, 1987. 